\title{
New techniques in endocytoscopy: submucosal injection heightens the visibility
}

Endocytoscopy involves the use of a light contact microscope capable of magnifying endoscopic images up to $\times 520$, allowing optical biopsy to the cellular level [ 1 , 2].

A 69-year-old patient had an 8-mm 0-Ila lesion located in the transverse colon. We performed colonoscopy using endocytoscopy (CF-H290ECl; Olympus, Tokyo, Japan) without distal attachment (-Video 1).

Magnifying narrow-band imaging (NBI) showed Japan NBI Expert Team classification type $2 \mathrm{~A}$ around the whole surface of the lesion ( Fig.1a) [3]. We then stained the lesion with $0.05 \%$ crystal violet. Magnifying chromoendoscopy re-

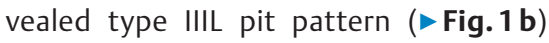
based on Kudo's classification [3].

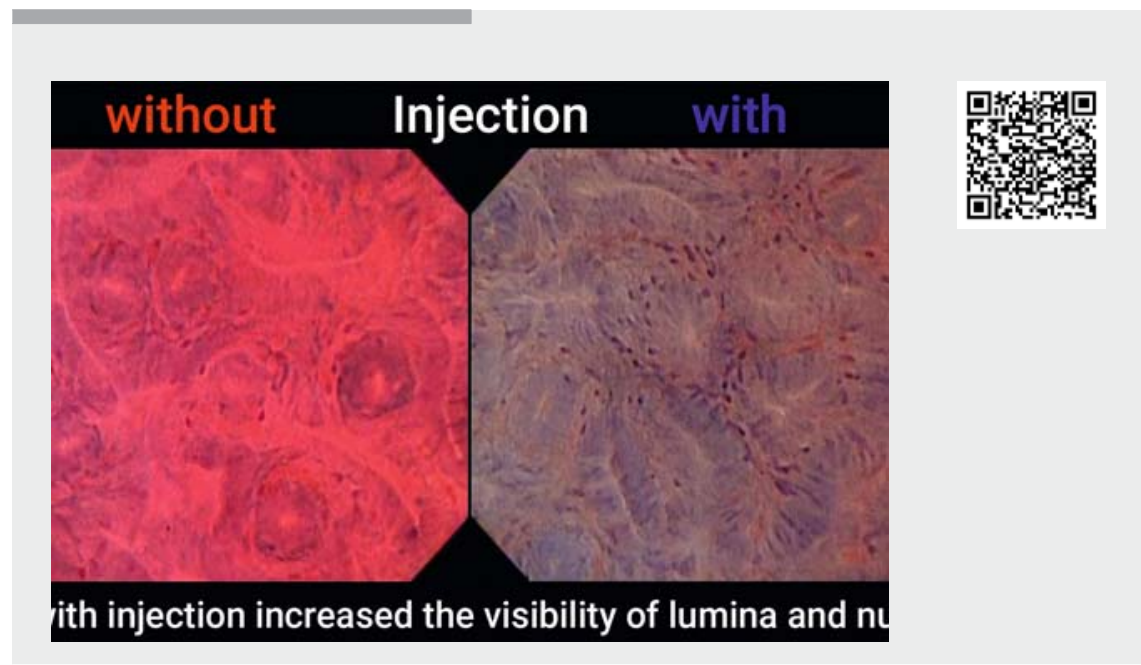

Video 1 Endocytoscopy for an 8-mm 0-Ila lesion, located in the transverse colon. Because of the lack of light, we performed submucosal injection. Submucosal injection increased visualization of the lesion at the cellular level. EC, endocytoscopy.
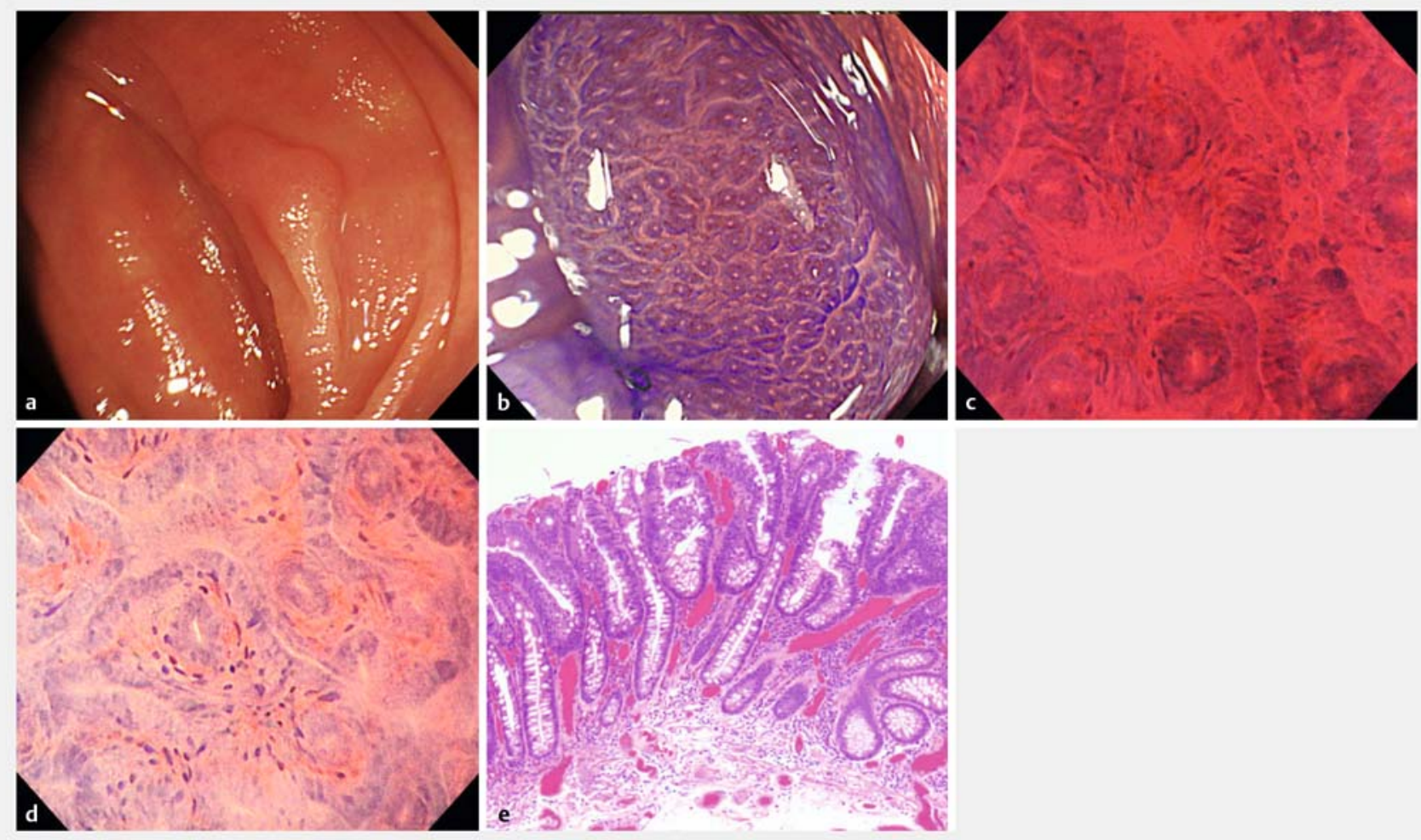

- Fig. 1 Endoscopic findings, treatment, and pathology. a An 8-mm 0-Ila lesion was located in the transverse colon. b Magnifying chromoendoscopy using crystal violet staining identified a type IIIL pit pattern. $\mathbf{c}$ The lesion was stained with methylene blue and endocytoscopic examination was performed $(\times 520)$. d Submucosal injection was performed and endocytoscopic examination was repeated. e The lesion was pathologically diagnosed as low grade adenoma. 
Subsequently, the lesion was stained with $1 \%$ methylene blue ( $\triangleright$ Fig. 1 c, $\times 520$ ). Diagnosis was made on the basis of $\mathrm{Ku}$ do's endocytoscopy classification [4]. However, it was difficult to evaluate lumina and nuclei, and we considered the lack of light to be the cause. Therefore, we performed submucosal injection of $0.9 \%$ saline under the lesion. In addition to the light reflected from the scope into the submucosal layer, submucosal injection led to a thickening of the submucosal layer, increasing the amount of light from the surroundings. The increased light intensity provided a clearer contrast to the background stroma and improved the visibility of the lumina and nuclei. The endocytoscopy image showed slitlike smooth lumina, and uniform and roundish nuclei ( $\mathbf{F i g . 1} \mathbf{1}$ ). Furthermore, because we did not find irregular and rough lumina and a large number of roundish nuclei, we diagnosed the lesion as EC2. Endoscopic mucosal resection was performed and histopathological findings showed low grade adenoma (॰ Fig. 1 e).

Submucosal injection improved cellular visualization because swelling of the submucosal layer with saline strengthened the contrast between stroma, lumina, and nuclei. This method may improve the diagnostic ability of endocytoscopy. Further assessment is necessary to evaluate the effectiveness.

Endoscopy_UCTN_Code_TTT_1AQ_2AJ

\section{Competing interests}

The authors declare that they have no conflict of interest.
The authors

Yoshiaki Kimoto ${ }^{1}$, Ken Ohata ${ }^{1}$, Maiko Takita ${ }^{1}$, Yohei Minato', Takashi Muramoto', Yoshiyuki Kayashima ${ }^{2,3}$, Ikuro Koba ${ }^{2}$

1 Department of Gastrointestinal Endoscopy, NTT Medical Center Tokyo, Tokyo, Japan

2 Department of Gastroenterology, Yamaga Chuo Hospital, Kumamoto, Japan

3 Department of Gastroenterology, Faculty of Medicine, Fukuoka University, Fukuoka, Japan

\section{Corresponding author}

\section{Ken Ohata, MD, PhD}

Department of Gastrointestinal Endoscopy, NTT Medical Center Tokyo, 5-9-22 Higashigotanda, Shinagawa-ku, Tokyo 141-8625, Japan

Fax: +81-3-34486541

ken.ohata1974@gmail.com

\section{References}

[1] Inoue H, Kazawa T, Sato Y et al. In vivo observation of living cancer cells in the esophagus, stomach, and colon using cathetertype contact endoscope, "Endo-Cytoscopy system". Gastrointest Endosc Clin N Am 2004; 14: 589-594

[2] Kudo T, Suzuki K, Kudo SE et al. Endocytoscopy for the differential diagnosis of colorectal low-grade adenoma: a novel possibility for the "resect and discard" strategy. Gastrointest Endosc 2020; 91: 676-683

[3] Sano Y, Tanaka S, Kudo SE et al. Narrowband imaging (NBI) magnifying endoscopic classification of colorectal tumors proposed by the Japan NBI Expert Team. Dig Endosc 2016; 28: 526-533

[4] Kudo SE, Misawa M, Wada Y et al. Endocytoscopic microvasculature evaluation is a reliable new diagnostic method for colorectal lesions (with video). Gastrointest Endosc 2015; 82: 912-923
Bibliography

Endoscopy 2021; 53: E245-E246

DOI 10.1055/a-1252-1963

ISSN 0013-726X

published online 23.9.2020

(c) 2020. Thieme. All rights reserved.

Georg Thieme Verlag KG, Rüdigerstraße 14,

70469 Stuttgart, Germany

\section{ENDOSCOPY E-VIDEOS}

https://eref.thieme.de/e-videos

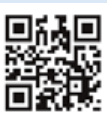

Endoscopy E-Videos is a free access online section, reporting on interesting cases and new

techniques in gastroenterological endoscopy. All papers include a high quality video and all contributions are freely accessible online.

This section has its own submission website at https://mc.manuscriptcentral.com/e-videos 